\title{
ANÁLISE DA RESPOSTA EM REGIME TRANSITÓRIO E PERMANENTE DE ALGORITMOS PARA FILTRAGEM DIGITAL UTILIZADOS EM RELÉS NUMÉRICOS: VELOCIDADE DE CONVERGÊNCIA, OVERSHOOT E SENSIBILIDADE EM RELAÇÃO A CONSTANTE DE TEMPO
}

\author{
Tatiane Martins Machado* \\ tatianemachado@mail.ufsm.br \\ Ghendy Cardoso Jr * \\ ghendyesmail.ufsm.br \\ Lenois Mariotto* \\ mariottoect.ufsm.br \\ * Universidade Federal de Santa Maria - UFSM \\ Centro de Estudos em Energia e Meio Ambiente - CEEMA \\ Bairro Camobi \\ CEP 97105-900 - Santa Maria RS
}

\section{RESUMO}

Analysis of the Transient and Steady State Performance of Digital Filtering Algorithms used in Numeric Relays: convergence speed, overshoot and time constant sensitivity

Este trabalho visa avaliar o desempenho de algoritmos de filtragem digital para relés numéricos em termos da capacidade de rejeição da componente DC, resposta transitória, exatidão, freqüência de amostragem e imunidade a ruídos. Além dos filtros clássicos de Fourier de ciclo completo e Cosseno também, são consideradas duas Transformadas Discretas de Fourier (TDF) modificadas. Os sinais de corrente utilizados nos testes foram gerados por equações matemáticas e por simulações computacionais em um sistema teste. Os resultados mostram que na maioria dos casos os filtros de Fourier modificados apresentam o melhor desempenho. Esses algoritmos exigem um maior esforço computacional, devido ao número de operações matemáticas

Artigo submetido em 18/11/2009 (Id.: 01080)

Revisado em 04/03/2010, 14/07/2010, 07/08/2010

Aceito sob recomendação do Editor Associado Prof. Julio Cesar Stacchini Souza envolvidas. No entanto, esse fato não causou aumento considerável no tempo de simulação.

PALAVRAS-CHAVE: Filtragem digital, relés de proteção, sinais exponenciais decrescentes.

\section{ABSTRACT}

This paper aims to assess the performance of digital filtering algorithms for numeric relays in terms of: ability to reject the DC offset component, transient response, accuracy, sampling frequency and noise immunity. The filters under investigation are the full cycle Fourier transform filters, cosine and two modified DFT methods. The current waveforms used during the tests were generated by mathematical equations and by computational simulation on a test system. The results show that the modified Fourier filters have the best performance, in most cases. These algorithms require a greater computational effort due to the number of mathematical operations involved. However, this fact has not caused considerable increase in simulation time.

KEYWORDS: Digital filtering, protective relaying, decaying 
DC offset.

\section{INTRODUÇÃO}

Os sinais de entrada dos relés de proteção contêm componentes indesejáveis que devem ser rejeitados de modo a reter somente o sinal de interesse. Dessa forma, os algoritmos responsáveis pela filtragem são de fundamental importância para o correto funcionamento do relé digital. Os requisitos de filtragem dependem do princípio de proteção e da aplicação. Alguns relés de distância, por exemplo, têm seu funcionamento baseado no cálculo da impedância aparente e, uma vez que a impedância é definida em uma determinada freqüência, os filtros dos relés de distância devem reter somente a componente do sinal nesta freqüência, que geralmente é a fundamental.

Exatidão e velocidade de convergência dos algoritmos de filtragem são essenciais para os relés de proteção, pois eles precisam efetuar decisões rápidas e confiáveis, mesmo na presença da componente DC e harmônicas de ordem superior.

Os sinais de corrente resultantes de uma falta ou de um distúrbio no sistema elétrico de potência podem conter uma componente unidirecional que decai exponencialmente no tempo, juntamente com as componentes senoidais. A componente DC é resultado da mudança instantânea do estado pré-falta para pós-falta (Stringer, 1997). A taxa de decaimento da componente DC depende da constante de tempo do sistema e pode ser determinada pela razão $X / R$ no ponto da falta. Como esta componente apresenta uma característica exponencial, ou seja, é um sinal não-periódico, seu espectro de freqüências apresenta uma faixa de freqüências relativamente grande (Sidhu et al., 2003). A componente DC exerce infuência direta na exatidão e na velocidade de convergência dos algoritmos utilizados para estimar os fasores (Yu \& Gu, 2001).

O projeto de filtros digitais e o estabelecimento de índices adequados para avaliar a eficiência dos algoritmos utilizados em relés numéricos, tem sido motivo de pesquisa, pois só assim, consegue-se verificar o porquê de uma possível falha do relé devido a erro de medição do algoritmo utilizado (Veloso, 2006; Miranda, 2005).

A Transformada Discreta de Fourier (TDF), ou filtro de Fourier, é o algoritmo mais utilizado para estimar os fasores da componente de frequiência fundamental em relés digitais de proteção. A TDF é capaz de remover harmônicas por meio de cálculos bastante simples. Embora diferentes janelas de dados tenham sido sugeridas, a Transformada Discreta de Fourier de Ciclo Completo (TDFCC) é a mais utilizada. A componente DC introduz erros significativos nos fasores estimados pela TDF, os quais não devem ser tolerados na maioria das aplicações envolvendo relés (Sidhu et al., 2003).

Assim como a TDF, os filtros Seno e Cosseno também são capazes de remover harmônicas por meio de cálculos simples. O filtro Cosseno de um ciclo é capaz de rejeitar a componente DC e todas as harmônicas, e tem uma característica muito parecida com o filtro passa-banda. O filtro Seno tem uma melhor atenuação para as altas freqüências e também rejeita todas as harmônicas. No entanto, o filtro Seno não é capaz de rejeitar a componente DC (Schweitzer \& Hou, 1993).

Muitas técnicas têm sido propostas no sentido de reduzir ou remover o efeito da componente DC nos fasores estimados. Sachdev \& Baribeau (1979) apresentaram um filtro baseado no método dos Mínimos Quadrados. Este algoritmo modela os parâmetros da componente DC como uma constante mais uma rampa linearmente declinante, utilizando os dois primeiros termos da expansão em série de Taylor. Segundo Sidhu et al. (2003), o desempenho deste algoritmo não é satisfatório quando a constante de tempo do sistema for muito pequena.

Benmouyal (1995) propôs o filtro digital mimic. Esse filtro só pode remover completamente a componente $\mathrm{DC}$, quando a constante de tempo do sistema for igual a constante de tempo considerada no algoritmo. Esta condição não é comum em um sistema de potência real, uma vez que a constante de tempo e a amplitude da componente DC são desconhecidas, pois são função da resistência de falta, posição da falta e ângulo da tensão no instante da falta ( $\mathrm{Yu} \& \mathrm{Gu}, 2001$ ). Ainda, segundo Phadke \& Thorp (1988), qualquer ruído presente no sinal da corrente tende a ser amplificado pelo circuito mimic.

Yu \& Gu (2001) apresentaram um algoritmo que faz uso de um filtro analógico passa-baixas capaz de remover harmônicas de ordem superior, e um filtro TDF modificado de ciclo completo (TDFCC) ou de meio ciclo para eliminar a componente DC . O algoritmo TDFCC modificado requer um ciclo mais três ou quatro amostras e o algoritmo TDF modificado de meio ciclo requer meio ciclo mais quatro amostras. Estas amostras adicionais aumentam o tempo de resposta do algoritmo quando comparado a TDF convencional. Portanto, caso a componente DC não esteja presente no sinal, a TDF convencional apresentará uma maior velocidade de resposta.

Sidhu et al. (2003) propuseram uma técnica para remover a componente DC dos fasores estimados pelo algoritmo TDF. O algoritmo é baseado na estimação da componente DC do sinal e pode se adaptar automaticamente ao sistema e às condições de falta. São usados dois filtros de Fourier de ciclo completo (TDFCC) em paralelo, um sintonizado na 
freqüência fundamental e outro, em uma freqüência superior a de corte do filtro passa-baixas. O objetivo do filtro sintonizado em uma freqüência mais elevada é determinar os parâmetros da componente DC.

Outro algoritmo TDF modificado foi proposto por Lee et al. (2008). Os autores propuseram um método que decompõe o processo da TDF convencional em uma TDF que utiliza amostras pares e outra TDF que utiliza amostras ímpares. O efeito da componente DC é eliminado usando a relação recursiva entre as saídas das TDFs com amostras pares e ímpares. O algoritmo apresenta resultados satisfatórios mesmo quando o sinal de entrada contém duas componentes DC com diferentes constantes de tempo.

Seguindo esta linha de pesquisa, este artigo visa avaliar o desempenho dos algoritmos de filtragem mais utilizados em relés digitais, juntamente com aqueles recentemente apresentados na literatura técnica. Os filtros analisados são o de Fourier de ciclo completo, Cosseno, TDF modificada proposta por Sidhu et al. (2003) e a TDF modificada proposta por Lee et al. (2008). Os algoritmos são avaliados em termos da capacidade de rejeição da componente DC, resposta transitória, exatidão, frequiência de amostragem e imunidade a ruídos.

\section{ALGORITMOS DE FILTRAGEM DIGITAL}

Os primeiros algoritmos desenvolvidos para relés digitais foram voltados para a proteção de linhas de transmissão. Como exemplo, se têm os algoritmos para relés de distância clássicos, os quais realizam o cálculo da impedância aparente por meio das amostras de tensão e corrente na freqüência fundamental. $\mathrm{O}$ desempenho desses algoritmos depende da exatidão das componentes de frequiência fundamental estimadas a partir das amostras do sinal. Se o sinal for puramente senoidal, todos os algoritmos de filtragem são capazes de estimar os fasores de maneira correta. A distinção entre os algoritmos surge quando componentes diferentes da freqüência fundamental estão presentes no sinal (Phadke \& Thorp, 1988).

\subsection{Filtro de Fourier de ciclo completo}

O filtro de Fourier de ciclo completo, ou Transformada Discreta de Fourier de ciclo completo (TDFCC), é um dos filtros mais utilizados em relés de proteção. A estimação da componente de frequiência fundamental é feita por meio da correlação entre as amostras de um ciclo do sinal a ser filtrado com as amostras de um ciclo dos sinais de referência, seno e cosseno, na freqüência fundamental (IEEE Tutorial Course, 1979).

Com $N$ amostras por ciclo, $(N / 2)$-1 harmônicas podem ser calculadas (Phadke \& Thorp, 1988). A $m$-ésima harmônica pode ser calculada por:

$$
\begin{aligned}
& I_{c}^{m}=\frac{2}{N} \sum_{n=0}^{N-1} i[n] \cos \left(n \frac{2 \pi}{N} m\right) \\
& I_{s}^{m}=\frac{2}{N} \sum_{n=0}^{N-1} i[n] \operatorname{sen}\left(n \frac{2 \pi}{N} m\right)
\end{aligned}
$$

onde $i[n]$ é a $n$-ésima amostra do sinal a ser filtrado. Quando $m$ for considerado igual a 1 , o algoritmo será referenciado como TDFCC de freqüência fundamental.

A magnitude e a fase da $m$-ésima harmônica são obtidas pelas equações (3) e (4), respectivamente.

$$
\begin{aligned}
\left|I^{m}\right| & =\sqrt{\left(I_{c}^{m}\right)^{2}+\left(I_{s}^{m}\right)^{2}} \\
\theta_{m} & =\tan ^{-1}\left(\frac{I_{s}^{m}}{I_{c}^{m}}\right)
\end{aligned}
$$

\subsection{Filtro cosseno}

Os coeficientes do filtro cosseno correspondem às amostras de um ciclo da forma de onda de uma cossenóide (Schweitzer \& Hou, 1993). A $m$-ésima harmônica pode ser determinada por (Wang, 1999):

$$
\begin{gathered}
I_{c}^{m}=\frac{2}{N} \sum_{n=0}^{N-1} i[n] \cos \left(n \frac{2 \pi}{N} m\right) \\
I_{s}^{m}=\frac{2}{N} \sum_{n=0}^{N-1} i[n-N / 4] \cos \left(n \frac{2 \pi}{N} m\right)
\end{gathered}
$$

\subsection{Filtro de Fourier modificado proposto por SIDHU et al. (2003)}

Sidhu et al. (2003) propuseram uma técnica que visa reduzir o efeito da componente DC nos fasores estimados por meio do filtro de Fourier de ciclo completo. A técnica é baseada na estimação da componente DC e pode se adaptar automaticamente às variações da constante de tempo da componente DC $(\tau)$.

O sinal de corrente, durante condições de falta, pode ser considerado como a combinação de uma componente DC e componentes senoidais. Admitindo que as componentes 
senoidais com freqüências superiores à $p$-ésima harmônica são eliminadas pelo filtro passa-baixas anti-aliasing, então, o sinal de corrente discreto visto pelo dispositivo de proteção é dado por:

$$
i[n]=I_{0} e^{-n T / \tau}+\sum_{k=1}^{p} I_{k} \operatorname{sen}\left(k \omega_{1} n T+\alpha_{k}\right)
$$

onde $I_{0}$ é a magnitude da componente DC, $\tau$ é a constante de tempo da componente DC, $I_{k}$ é a magnitude da $k$-ésima harmônica, $\omega_{1}$ é a freqüência angular fundamental, $\alpha_{k}$ é o ângulo de fase da $k$-ésima harmônica, Té o período de amostragem e $n e ́$ o índice da $n$-ésima amostra.

A componente de freqüência fundamental do sinal representado pela equação (7) pode ser calculada por meio do algoritmo TDFCC (equações (1) e (2)), considerando $m=1$. O algoritmo TDFCC de freqüência fundamental também pode ser expresso como:

$$
I_{T D F C C}=\frac{2}{N} \sum_{n=0}^{N-1} i[n] j e^{-j \frac{2 \pi}{N} n}
$$

onde $I_{T D F C C}$ é a saída do filtro TDFCC na frequiência fundamental correspondente ao sinal de entrada total (harmônicas e componente DC).

Como as harmônicas são totalmente eliminadas pelo TDFCC, somente as componentes de freqüência fundamental e DC aparecerão na saída do filtro, como mostrado na equação (9).

$$
I_{T D F C C}=I_{T D F C C}^{1}+I_{T D F C C}^{d c}
$$

onde $I^{1} T D F C C$ é a componente de frequiência fundamental e $I^{d c} T D F C C$ é a componente DC.

Portanto, o fasor de freqüência fundamental é dado por:

$$
I_{T D F C C}^{1}=I_{T D F C C}-I_{T D F C C}^{d c}
$$

Dessa forma, a componente de freqüência fundamental pode ser encontrada, se o termo $I_{T D F C}^{d c} C$ for conhecido. Esse termo representa a resposta do TDFCC quando se considera somente a componente DC $\left(I_{0} e^{-n T / \tau}\right)$ como entrada. Essa resposta é definida na equação (12).

$$
I_{T D F C C}^{d c}=\frac{2}{N} \sum_{n=0}^{N-1} I_{0} e^{-n T / \tau} \cdot j e^{-j \frac{2 \pi}{N} n}
$$

$$
I_{T D F C C}^{d c}=\frac{2}{N} \cdot j I_{0}\left(\frac{1-e^{-N T / \tau}}{1-e^{-T / \tau} \cdot e^{-j \frac{2 \pi}{N}}}\right)
$$

Note que $I^{d c} T D F C C$ é função da magnitude e da constante de tempo da componente DC. Portanto, é necessário utilizar um método capaz de encontrar esses parâmetros.

A técnica proposta por Sidhu et al. (2003) é baseada no fato de que o filtro passa-baixas anti-aliasing elimina as componentes de alta freqüência e, portanto, componentes com freqüências bem mais altas que a freqüência de corte não estão presentes no sinal de saída do filtro passa-baixas. Dessa forma, foi proposto o projeto de outro filtro TDFCC (filtro 2) sintonizado em uma frequiência bem mais alta que a frequiência de corte do filtro passa-baixas pois, assim, somente a componente DC afetará a saída desse filtro. O filtro 2 e o filtro de freqüência fundamental operam em paralelo.

Considerando que o filtro 2 está sintonizado na $m$-ésima harmônica, e que a frequiência dessa harmônica esteja bem acima da freqüência de corte do filtro passa-baixas e abaixo da metade da freqüência de amostragem, então a saída do filtro é dada por:

$$
\begin{gathered}
I_{T D F C C}^{m}=\frac{2}{N} \sum_{n=0}^{N-1} I_{0} e^{-n T / \tau} j\left(e^{-j n \frac{2 \pi}{N} m}\right) \\
I_{T D F C C}^{m}=\frac{2}{N} j I_{0}\left(\frac{1-e^{-N T / \tau}}{1-e^{-T / \tau} \cdot e^{-j m \frac{2 \pi}{N}}}\right)
\end{gathered}
$$

onde $I_{T D F C C}^{m}$ é a saída do algoritmo TDFCC sintonizado na $m$-ésima harmônica.

A equação (14) pode ser escrita em função da parte real $(R)$ e imaginária $(I)$, como mostrado nas equações (15) e (16), respectivamente. Por simplicidade, a expressão $e^{-T / \tau}$ será considerada como $E$.

$$
\begin{array}{r}
R=\frac{2}{N}\left(\frac{I_{0}\left(1-E^{N}\right) E \operatorname{sen}(\omega m T)}{1+E^{2}-2 E \cos (\omega m T)}\right) \\
I=\frac{2}{N}\left(\frac{I_{0}\left(1-E^{N}\right)(1-E \cos (\omega m T))}{1+E^{2}-2 E \cos (\omega m T)}\right)
\end{array}
$$

Das equações (15) e (16), $E$ e $(2 / N) I_{0}\left(1-E^{N}\right)$ podem ser obtidos:

$$
E=\frac{R}{R \cos (\omega m T)+I \operatorname{sen}(\omega m T)}
$$




$$
\frac{2}{N} I_{0}\left(1-E^{N}\right)=\frac{R\left(1+E^{2}-2 E \cos (\omega m T)\right)}{E \operatorname{sen}(\omega m T)}
$$

Substituindo os valores obtidos nas equações (17) e (18) na equação (12), pode-se determinar a saída do TDFCC devido a componente DC. Dessa forma, o fasor de freqüência fundamental é deterninado por meio da equação (10).

O parâmetro $m$ foi escolhido a partir de várias simulações, de modo que o filtro apresentasse os melhores resultados. O valor de $m$ foi assumido como sendo 31, 47 e 59, para sinais de teste com freqüências de amostragem de $3840 \mathrm{~Hz}, 5760$ $\mathrm{Hz}$ e $7200 \mathrm{~Hz}$, respectivamente.

\subsubsection{Filtro de Fourier modificado proposto por LEE et al. (2008)}

O algoritmo proposto por Lee et al. (2008) utiliza o mesmo princípio do algoritmo proposto por Sidhu et al. (2003), ou seja, a componente de frequiência fundamental pode ser estimada se a resposta da TDFCC devido à componente DC for conhecida. Dessa forma, o fasor de freqüência fundamental é obtido por meio da equação (10).

Lee et al. (2008) propuseram uma nova maneira de obter a resposta da TDFCC devido à componente $\mathrm{DC}\left(I^{d c} T D F C C\right)$.

A TDFCC mostrada na equação (19), pode ser decomposta em duas partes: uma que leva em consideração somente as amostras pares e outra, as amostras ímpares, ou seja:

$$
\begin{gathered}
I_{T D F C C}=\frac{2}{N} \sum_{n=0}^{N-1} i[n] e^{-j \frac{2 \pi}{N} n} \\
I_{T D F C C}=\frac{2}{N} \sum_{n=0}^{N / 2-1} i[2 n]\left(e^{-j \frac{2 \pi}{N} 2 n}\right)+ \\
\frac{2}{N} \sum_{n=0}^{N / 2-1} i[2 n+1]\left(e^{-j \frac{2 \pi}{N}(2 n+1)}\right) \\
I_{T D F C C}=I_{T D F C C}^{\text {par }}+I_{T D F C C}^{i m p a r}
\end{gathered}
$$

A saída da TDFCC de amostras pares é:

$$
I_{T D F C C}^{p a r}=\frac{1}{2} I_{1} e^{j \phi_{1}}+\frac{2}{N} \cdot I_{0} \cdot \frac{1-E^{N}}{1-\left(E e^{-j \frac{2 \pi}{N}}\right)^{2}}
$$

onde $I_{1}$ e $\Phi_{1}$ são a amplitude e a fase da componente de frequiência fundamental, respectivamente. $\mathrm{O}$ termo
$E$ representa a expressão $e^{-T / \tau}$ e $I_{0}$ é a amplitude da componente DC. Dessa forma, o primeiro termo de $I^{p a r} T D F C C$ equivale a uma parcela da componente de frequiência fundamental e o segundo, representa uma parcela da componente DC.

A saída da TDFCC de amostras ímpares é dada por:

$$
I_{T D F C C}^{i m p a r}=\frac{1}{2} I_{1} e^{j \phi_{1}}+\frac{2}{N} E \cdot e^{-j \frac{2 \pi}{N}} \cdot I_{0} \cdot \frac{1-E^{N}}{1-\left(E e^{-j \frac{2 \pi}{N}}\right)^{2}}
$$

Da mesma forma que no caso anterior, o primeiro termo de $I^{\text {impar }}{ }_{T D F C C}$ representa uma parcela da componente de frequiência fundamental e o segundo termo, uma parcela da componente DC.

Como se deseja obter somente a resposta do filtro devido a componente DC, é necessário eliminar a componente fundamental. Isso é feito por meio da diferença entre as equações (22) e (23).

$$
I_{T D F C C}^{\text {par }}-I_{T D F C C}^{i m p a r}=\frac{2}{N} \cdot I_{0} \cdot \frac{1-E^{N}}{1+E e^{-j \frac{2 \pi}{N}}}
$$

A equação (24) apresenta a diferença entre a saída da TDFCC de amostras pares e ímpares . Escrevendo a equação (24) em função da parte real $(R)$ e imaginária $(I)$, tem-se:

$$
R=\frac{2}{N} I_{0} \frac{1-E^{N}}{1+E^{2}+2 E \cos (2 \pi / N)}(1+E \cos (2 \pi / N))
$$

$$
I=\frac{2}{N} I_{0} \frac{1-E^{N}}{1+E^{2}+2 E \cos (2 \pi / N)} \operatorname{Esen}(2 \pi / N)
$$

A partir das equações (25) e (26), se pode obter $E$ :

$$
E=\frac{I}{R \operatorname{sen}(2 \pi / N)-I \cos (2 \pi / N)}
$$

Substituindo a equação (27) na equação (24), é possível determinar a resposta do TDFCC de freqüência fundamental devido a componente DC:

$$
I_{T D F C C}^{d c}=\left(I_{T D F C C}^{p a r}-I_{T D F C C}^{i m p a r}\right) \cdot \frac{\left(1+E e^{-j \frac{2 \pi}{N}}\right)}{\left(1-E e^{-j \frac{2 \pi}{N}}\right)}
$$




\subsubsection{Portanto, o fasor de freqüência fundamental pode ser determinado pela substituição da equação (28) na equação (10).}

Um fator muito importante para a análise das respostas dos filtros diz respeito ao comprimento da janela de dados. Todos os filtros fazem uso de uma janela de dados com $\mathrm{N}$ amostras, sendo que, quando uma nova amostra se torna disponível, esta é incluída nos cálculos e a mais antiga é descartada (Phadke \& Thorp, 1988).

Quando uma falta ocorre, a janela de dados passa a ser alimentada com valores relativos ao período pré-falta e pós-falta, permanecendo nessa situação até que todos os dados antigos sejam eliminados da janela. Durante esse período, os fasores estimados pelos filtros não tem significado algum e nenhuma decisão deve ser tomada pelo relé de proteção (Phadke \& Thorp, 1988). Segundo Schweitzer \& Hou (1993), o filtro está em um período transitório. Quando a janela de dados passar a incluir somente os dados relativos ao período durante a falta, o filtro passa ao estado estável.

Portanto, como uma janela de dados mais longa necessita de mais tempo para ser preenchida com amostras referentes ao período da falta, é evidente que uma decisão mais rápida pode ser obtida com algoritmos que utilizam janelas mais curtas. No entanto, a habilidade de um algoritmo rejeitar sinais com frequiências diferentes da fundamental é função do comprimento da janela de dados. Em outras palavras, há uma relação inversa entre velocidade e precisão do relé (Phadke \& Thorp, 1988).

\section{AVALIAÇÃO DE DESEMPENHO}

Para verificar a eficiência dos filtros, foram utilizados índices de desempenho propostos por Benmouyal (1995) e por Balamourougan \& Sidhu (2006). O primeiro propõe índices que avaliam a resposta do filtro em regime permanente, o percentual de overshoot na resposta de saída e a sensibilidade dos filtros com a variação da constante de tempo $(\tau)$. O segundo, propõe um índice que avalia a velocidade de convergência da resposta dos filtros. Os índices foram utilizados para avaliar tanto a resposta de magnitude quanto a resposta de fase dos fasores estimados pelos filtros.

Considere que $i(t)$ seja um sinal cuja amplitude e ângulo são representados por amp e ang, respectivamente. Ao aplicar esse sinal à entrada de um filtro digital sintonizado na freqüência $m$, obtém-se como resposta a magnitude e o ângulo estimados, denominados por $I^{m}(t)$ e $\theta^{m}(t)$, respectivamente. Assim, o índice que avalia a resposta de magnitude e da fase dos filtros em regime permanente são dados pelas equações (29) e (30), respectivamente.

$$
\begin{aligned}
& I D_{1}(\tau)=\int_{T_{0}}^{N T}\left[a m p-I^{m}(t)\right]^{2} d t \\
& I D_{2}(\tau)=\int_{T_{0}}^{N T}\left[\operatorname{ang}-\theta^{m}(t)\right]^{2} d t
\end{aligned}
$$

onde $T_{0}$ é o instante em que $I^{m}(t)$ e $\theta^{m}(t)$ atingem o valor amp e ang, respectivamente, e, NT representa a duração de $\mathrm{N}$ ciclos, contados a partir do instante zero, que se deseja analisar (escolheu-se arbitrariamente $N$ igual a 6 ciclos). Estes índices indicam o quanto a resposta oscila em torno do valor desejado, sendo que $I D_{1}$ e $I D_{2}$ próximos a zero indicam que o filtro é livre de oscilação em regime permanente, ou seja, o filtro apresenta uma maior tolerância para com a componente exponencial DC.

O percentual de overshoot na resposta de magnitude e de fase é dado pelas equações (31) e (32), respectivamente.

$$
\begin{aligned}
I D_{3}(\tau) & =\left(\max \left[I^{m}(t)-a m p\right]\right) \cdot 100 \\
I D_{4}(\tau) & =\left(\max \left[\theta^{m}(t)-a n g\right]\right) \cdot 100
\end{aligned}
$$

As equações (33) e (34) avaliam a velocidade de convergência da resposta de magnitude e de fase, respectivamente.

$$
\begin{aligned}
& I D_{5}(\tau)=\frac{N_{a m p}-N}{N} \\
& I D_{6}(\tau)=\frac{N_{a n g}-N}{N}
\end{aligned}
$$

onde $N_{a m p}$ é o número de amostras necessárias para a resposta de magnitude convergir, $N_{a n g}$ é o número de amostras necessárias para a resposta de fase convergir e, $\mathrm{N}$ representa o número de amostras por ciclo do sinal de entrada $i(t)$.

No caso da resposta de magnitude, espera-se que os filtros forneçam como resposta o valor amp, no entanto, existem instantes em que a resposta possui uma pequena variação em torno desse valor. Como essa variação, muitas vezes, se deve a erros numéricos gerados pelo computador e pelo software de simulação, adotou-se uma faixa de erro aceitável. Assim, considera-se que a convergência foi alcançada quando a resposta se encontrar dentro dessa faixa de valores durante dois ciclos consecutivos. O erro de convergência admitido, tanto para a magnitude quanto para a fase, foi de $\pm 0.1 \%$. Note que quanto maiores forem os índices $I D_{5}$ e $I D_{6}$, mais baixa será a velocidade de convergência. 
Os índices apresentados são calculados para um valor particular da constante de tempo $\tau$. Porém, em casos reais é difícil estimar o valor de $\tau$, já que o mesmo é função de diversos fatores relacionados à falta. Deste modo, é importante analisar a sensibilidade dos filtros para diversos valores de $\tau$. Isso é feito por meio da média de cada um dos índices de desempenho, quando calculados para constantes de tempo variando de 0,5 a 5 ciclos, como mostrado na equação (35).

$$
M\left(I D_{i}\right)=\frac{I D_{i}(0,5)+\sum_{\tau=1}^{5} I D_{i}(\tau)}{6}, i=1,2, \ldots, \text { ou } 6
$$

\section{RESULTADOS DAS SIMULAÇÕES}

Dois tipos de sinais de teste foram utilizados para avaliar o comportamento dos filtros: sinais analíticos e sinais obtidos a partir de simulações no programa ATP. Os sinais analíticos garantem a presença de determinadas ordens harmônicas e constantes de tempo nos sinais de entrada. Esses permitem avaliar os filtros com base na variação da componente DC e capacidade de rejeição de harmônicas. Já os sinais gerados pelo programa ATP reproduzem situações mais reais, uma vez que diversos tipos de curto-circuitos podem ser simulados, variando-se o tempo de inserção da falta, bem como o ponto de ocorrência desta.

Para analisar a influência da frequiência de amostragem no comportamento dos filtros, os sinais foram amostrados nas frequiências de $3840 \mathrm{~Hz}, 5760 \mathrm{~Hz}$ e $7200 \mathrm{~Hz}$, isto é, 64, 96 e 120 amostras por ciclo em um sistema de $60 \mathrm{~Hz}$, respectivamente. Em todos os casos, o sinal de entrada foi pré-processado por um filtro passa-baixas Butterworth de $2^{\mathrm{a}}$ ordem com frequiência de corte de $540 \mathrm{~Hz}$, de modo a remover harmônicas de ordem elevada.

\subsection{Sinais analíticos para teste}

Os sinais analíticos foram divididos em três tipos:

- Sinais com harmônicas e uma componente DC;

- Sinais com harmônicas e duas componentes DC;

- Sinais com harmônicas, duas componentes DC e ruídos aleatórios.

A utilização de sinais de teste com múltiplas componentes DC se deve ao fato de que, quando a corrente de falta, constituída por componentes senoidais e uma componente
DC (ou componente DC primária), é medida pelo Transformador de Corrente (TC), uma nova componente DC é gerada, sendo esta chamada de componente DC secundária (Lee et al, 2008).

A máxima ordem harmônica considerada nos sinais de teste foi 30, de acordo com Yu \& Gu (2001). Uma vez que a magnitude da componente DC varia com a constante de tempo e com o ângulo da tensão no instante da inserção da falta, dois diferentes ângulos foram considerados $\alpha=$ $10^{\circ}, 45^{\circ}$ para seis diferentes constantes de tempo $(\tau=$ $0.5,1,2,3,4$ e 5 ciclos).

\subsubsection{Sinais com harmônicas e uma componente DC}

O sinal de teste utilizado neste caso é dado por:

$$
i_{1}(t)=\left[\sum_{n=1}^{30} \frac{50}{n} \cos (n \omega t+n \alpha)\right]-50 \cos \alpha \cdot e^{-\frac{t}{\tau}}
$$

A Figura 1 apresenta alguns desses sinais com constantes de tempo iguais a 0,5 e 5 ciclos.

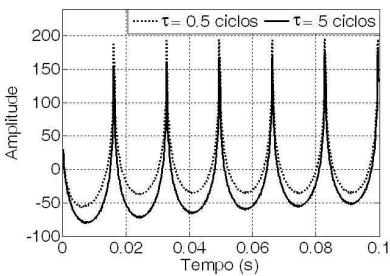

(a) $\alpha=10^{\circ}$

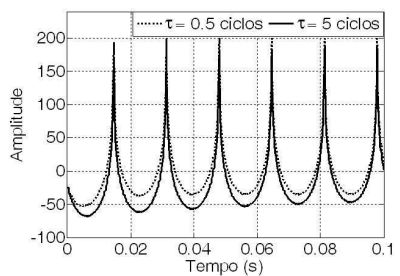

(b) $\alpha=45^{\circ}$
Figura 1: Sinais de teste contendo harmônicas até a ordem 30 e uma componente DC.

As respostas dos filtros no domínio do tempo, para $\alpha=10^{\circ}$, são apresentadas na Figura 2. Os resultados consideram os sinais amostrados em $3840 \mathrm{~Hz}$.

Todos os filtros exibiram boa resposta quando submetidos a sinais de entrada livres da componente DC, sendo que o TDFDC apresentou o melhor desempenho nesse caso.

Para sinais contendo a componente DC, verifica-se, pela Figura 2, que a oscilação da resposta é proporcional ao aumento da constante de tempo do sinal, sendo que, para $\tau=5$ ciclos, somente os filtros de Fourier modificados convergem. No entanto, a oscilação que ocorre para $\tau=5$ ciclos é de baixa amplitude quando comparada ao overshoot para $\tau=0,5$ ciclos. Como o overshoot influencia no cálculo da resposta em regime permanente, os índices $I D 1$ e ID2, muitas vezes, 
apresentam valores altos para constantes de tempo baixas, não correspondendo ao fato observado na Figura 2.
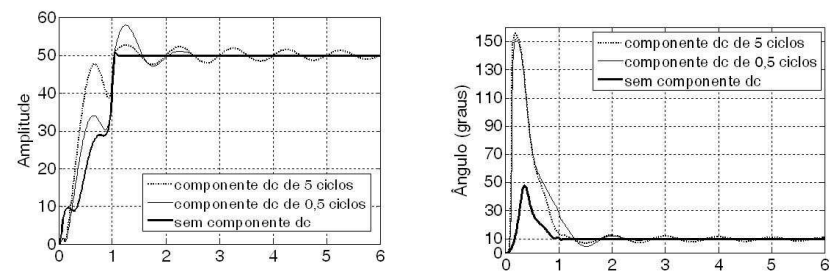

(a) TDFCC
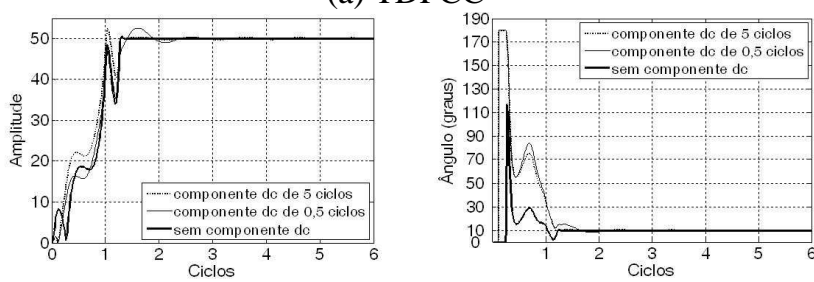

(b) Cosseno
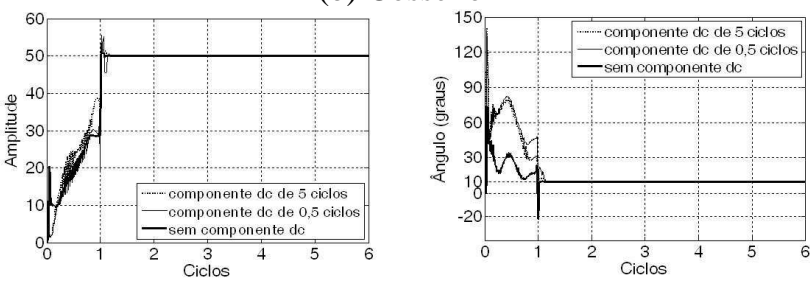

(c) TDF modificada proposta por Lee et al. (2008)
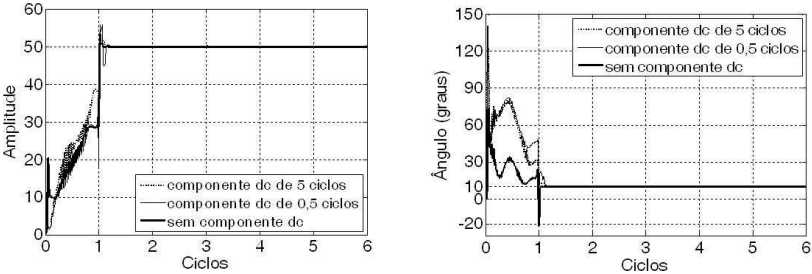

(d) TDF modificada proposta por Sidhu et al. (2003)

Figura 2: Resposta dos filtros, no domínio do tempo, para sinais baseados na equação (36), com ângulo de inserção de falta $\alpha=10^{\circ}$.

Quanto aos índices $I D 1$ e ID2, somente seus valores médios foram considerados, os quais, na realidade, envolvem tanto a oscilação como o overshoot. Uma vez que ambos devem ser mínimos, é desejado que $M\left(I D_{1}\right)$ e $M\left(I D_{2}\right)$ assumam os menores valores possíveis. Esse mesmo procedimento foi aplicado aos demais casos que serão apresentados posteriormente, uma vez que esse fato (influência do overshoot no cálculo de ID1 e ID2) foi observado em todas as simulações.

Por meio dos índices médios, $M\left(I D_{1}\right)$ e $M\left(I D_{2}\right)$, observou-se que os filtros de Fourier modificados apresentaram as menores oscilações da resposta de magnitude, e o filtro cosseno, da resposta de fase.

A Figura 2 mostra que o overshoot da resposta de fase é bastante elevado e ocorre sempre em menos de um ciclo, ou seja, quando a janela de dados ainda não está completa. Conforme mencionado anteriormente, nesse período, o filtro está em um estado transitório e nenhuma decisão será tomada pelo dispositivo de proteção. Assim, os altos índices ID4 não influenciarão diretamente na escolha do melhor filtro.

Já o overshoot da resposta de magnitude ocorre, na maioria dos casos, depois de um ciclo, fazendo com que o índice ID3 tenha maior importância. De maneira geral, o aumento da constante de tempo implica na redução do overshoot da resposta de magnitude.

O filtro cosseno é o que fornece a resposta de magnitude com menor overshoot. No entanto, como se pode observar na Figura 2, os filtros de Fourier modificados são os únicos que apresentam overshoot antes ou muito próximo a um ciclo. Como os relés de proteção devem operar rapidamente, é desejado que os erros gerados pelos filtros ocorram em um tempo que implique em um menor atraso do relé. Esse fato faz com que os filtros de Fourier modificados apresentem vantagens sobre o filtro cosseno, o qual apresenta overshoot em torno de 1 ciclo e meio.

Quanto à estabilização da resposta, os filtros tendem a não convergir à medida que a constante de tempo é aumentada, ou seja, quanto maior a constante de tempo, maior o tempo de oscilação da resposta em torno do valor correto. Os índices ID5 e ID6 comprovam esse fato. Foi utilizada a designação "Grande" para indicar que o filtro não convergiu em 6 ciclos (período de tempo analisado). Somente os filtros de Fourier modificados convergem para sinais com quaisquer constantes de tempo e, ainda, a velocidade de convergência praticamente não é alterada com o aumento da constante de tempo.

De acordo com as Tabelas 1 e 2, os filtros mais afetados pela variação da freqüência de amostragem $(3840 \mathrm{~Hz}, 5760 \mathrm{~Hz}$ e $7200 \mathrm{~Hz}$ ) são os de Fourier modificados. Esses apresentaram um melhor desempenho para a taxa de amostragem de 3840 $\mathrm{Hz}$.

Onde:

A - Filtro de Fourier de ciclo completo

B-Filtro Cosseno

C - Filtro de Fourier modificado proposto por Lee et al. (2008)

D - Filtro de Fourier modificado proposto por Sidhu et al. (2003) 
Tabela 1: Índices médios de desempenho para o sinal de teste da equação (36) para $\alpha=10^{\circ}$.

\begin{tabular}{|c|c|c|c|c|c|c|c|}
\hline Filtro & $\mathbf{N}$ & $M\left(I D_{1}\right)$ & $M\left(I D_{2}\right)$ & $M\left(I D_{3}\right)$ & $M\left(I D_{4}\right)$ & $M\left(I D_{5}\right)$ & $M\left(I D_{6}\right)$ \\
\hline \multicolumn{8}{|c|}{$\alpha=10^{\mathbf{o}}$} \\
\hline \multirow{3}{*}{ A } & 64 & 0,2691 & 0,2354 & 10,428 & 1446,3 & Grande & Grande \\
\hline & 96 & 0,2688 & 0,2352 & 10,425 & 1462,6 & Grande & Grande \\
\hline & 120 & 0,2688 & 0,2352 & 10,425 & 1469,4 & Grande & Grande \\
\hline \multirow{3}{*}{ B } & 64 & 0,1495 & 0,0083 & 4,6419 & 1700,0 & Grande & Grande \\
\hline & 96 & 0,1446 & 0,0068 & 4,4493 & 1700,0 & Grande & Grande \\
\hline & 120 & 0,1428 & 0,0062 & 4,3817 & 1700,0 & Grande & Grande \\
\hline \multirow{3}{*}{ C } & 64 & 0,0173 & 0,0262 & 9,1233 & 1298,6 & 0,2031 & 0,2708 \\
\hline & 96 & 0,0207 & 0,1533 & 12,654 & 1342,1 & 0,2083 & 0,2743 \\
\hline & 120 & 0,0265 & 0,0977 & 14,837 & 1353 & 0,2042 & 0,2736 \\
\hline \multirow{3}{*}{ D } & 64 & 0,0173 & 0,0262 & 9,1233 & 1298,6 & 0,2031 & 0,2708 \\
\hline & 96 & 0,0207 & 0,1533 & 12,654 & 1342,1 & 0,2083 & 0,2743 \\
\hline & 120 & 0,0265 & 0,0977 & 14,837 & 1353 & 0,2042 & 0,2736 \\
\hline
\end{tabular}

Como os demais filtros não tiveram variação de desempenho significativa com a alteração da freqüência de amostragem,conclui-se que, nesse caso o aumento do número de amostras por ciclo não traz grandes vantagens. Ao contrário, aumenta o esforço computacional, já que a quantidade de amostras a serem processadas aumenta.

Com relação ao ângulo da tensão no instante da inserção da falta, $\alpha$, observou-se que todos os filtros sofrem influência. Porém, os comportamentos não seguem um padrão, ou seja, alguns filtros melhoraram seu desempenho quando $\alpha=45^{\circ}$, enquanto outros se comportaram de maneira inversa.

\subsubsection{Sinais com harmônicas e duas componentes DC}

A corrente de falta é composta por componentes senoidais e uma componente DC (aqui denominada de componente DC primária). No entanto, o TC, ao receber esse sinal, introduz uma nova componente DC, sendo esta denominada de componente DC secundária. Esta surge devido as perdas no núcleo, indutância de magnetização e carga do TC (Horowitz \& Phadke, 1995).

Para avaliar o comportamento dos filtros na presença de múltiplas componentes DC, o seguinte sinal de teste foi utilizado:
Tabela 2: Índices médios de desempenho para o sinal de teste da equação (36) para $\alpha=45^{\circ}$.

\begin{tabular}{|c|c|c|c|c|c|c|c|}
\hline Filtro & $\mathrm{N}$ & $M\left(I D_{1}\right)$ & $M\left(I D_{2}\right)$ & $M\left(I D_{3}\right)$ & $M\left(I D_{4}\right)$ & $M\left(I D_{5}\right)$ & $M\left(I D_{6}\right)$ \\
\hline \multicolumn{8}{|c|}{$\alpha=\mathbf{4 5}^{\mathbf{0}}$} \\
\hline \multirow{3}{*}{$\mathbf{A}$} & 64 & 0,1657 & 0,1390 & 8,2091 & 300,0 & Grande & Grande \\
\hline & 96 & 0,1660 & 0,1389 & 8,2073 & 300,0 & Grande & Grande \\
\hline & 120 & 0,1662 & 0,1389 & 8,2065 & 300,0 & Grande & Grande \\
\hline \multirow{3}{*}{ B } & 64 & 0,2057 & 0,0053 & 2,1653 & 300,0 & Grande & Grande \\
\hline & 96 & 0,1943 & 0,0044 & 2,2107 & 300,0 & Grande & Grande \\
\hline & 120 & 0,1900 & 0,0041 & 2,2263 & 300,0 & Grande & Grande \\
\hline \multirow{3}{*}{ C } & 64 & 0,0327 & 0,9205 & 12,182 & 299,9 & 0,1406 & 0,1979 \\
\hline & 96 & 0,0786 & 0,7316 & 31,730 & 300,1 & 0,1476 & 0,1927 \\
\hline & 120 & 0,0575 & 0,8285 & 26,286 & 300,0 & 0,1444 & 0,1861 \\
\hline \multirow{3}{*}{ D } & 64 & 0,0327 & 0,9205 & 12,182 & 300,0 & 0,1406 & 0,1979 \\
\hline & 96 & 0,0786 & 0,7316 & 31,730 & 300,0 & 0,1476 & 0,1927 \\
\hline & 120 & 0,0575 & 0,8285 & 26,286 & 300,0 & 0,1444 & 0,1861 \\
\hline
\end{tabular}

$$
\begin{aligned}
i_{2}(t)=\left[\sum_{n=1}^{30} \frac{50}{n} \cos (n \omega t+n \alpha)\right] & -55 \cos \alpha \cdot e^{-\frac{t}{\tau}} \\
& +5 \cos \alpha \cdot e^{-\frac{t}{\tau_{s}}}
\end{aligned}
$$

onde $\tau_{s}$ é a constante de tempo secundária Esta será de 20 ciclos, conforme Lee et al. (2008).

A Figura 3 mostra os sinais de teste, baseados na equação (37), com constantes de tempo primárias iguais a 0.5 e 5 ciclos.

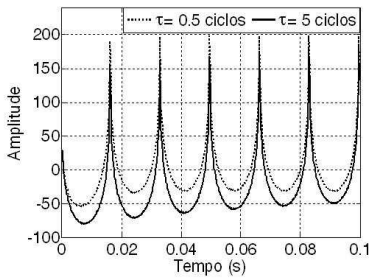

(a) $\alpha=10^{\circ}$

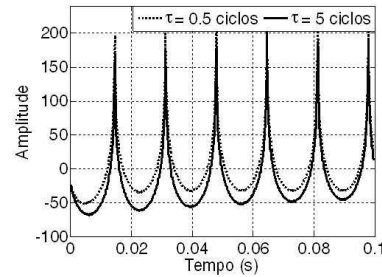

(b) $\alpha=45^{\circ}$
Figura 3: Sinais de teste contendo harmônicas até a ordem 30 e duas componentes DC.

As Tabela 3 e 4 apresentam as respostas dos filtros, no domínio do tempo, considerando a frequiência de amostragem de $3840 \mathrm{~Hz}$ e $\alpha=10^{\circ}$ e $45^{\circ}$, respectivamente.

De maneira geral, a inserção de uma nova componente DC no sinal de teste ocasionou um aumento nos índices que avaliam 
o desempenho dos filtros. No entanto, o comportamento das respostas em relação à variação da constante de tempo primária não foi alterado, ou seja, a oscilação da resposta permaneceu proporcional ao aumento da constante de tempo, e a velocidade de convergência e overshoot, inversamente proporcionais ao aumento de $\tau$.

Tabela 3: Índices médios de desempenho para o sinal de teste da equação (37) para $\alpha=10^{\circ}$.

\begin{tabular}{|c|c|c|c|c|c|c|c|}
\hline Filtro & $\mathbf{N}$ & $M\left(I D_{1}\right)$ & $M\left(I D_{2}\right)$ & $M\left(I D_{3}\right)$ & $M\left(I D_{4}\right)$ & $M\left(I D_{5}\right)$ & $M\left(I D_{6}\right)$ \\
\hline \multicolumn{8}{|c|}{$\alpha=10^{\mathbf{0}}$} \\
\hline \multirow{3}{*}{ A } & 64 & 0,3139 & 0,2713 & 11,323 & 1444,6 & Grande & Grande \\
\hline & 96 & 0,3137 & 0,2712 & 11,321 & 1461,0 & Grande & Grande \\
\hline & 120 & 0,3136 & 0,2711 & 11,320 & 1467,8 & Grande & Grande \\
\hline \multirow{3}{*}{ B } & 64 & 0,1547 & 0,0099 & 4,7487 & 1700,0 & Grande & Grande \\
\hline & 96 & 0,1491 & 0,0081 & 4,5578 & 1700,0 & Grande & Grande \\
\hline & 120 & 0,1470 & 0,0075 & 4,4908 & 1700,0 & Grande & Grande \\
\hline \multirow{3}{*}{ C } & 64 & 0,0203 & 0,0290 & 9,6042 & 1297,8 & 0,2031 & 0,7266 \\
\hline & 96 & 0,0236 & 0,1616 & 13,188 & 1340,6 & 0,2083 & 0,7266 \\
\hline & 120 & 0,0294 & 0,1049 & 15,344 & 1351,1 & 0,2056 & Grande \\
\hline \multirow{3}{*}{ D } & 64 & 0,0203 & 0,0290 & 9,6042 & 1297,8 & 0,2031 & 0,7266 \\
\hline & 96 & 0,0203 & 0,0290 & 9,6042 & 1297,8 & 0,2083 & 0,7266 \\
\hline & 120 & 0,0294 & 0,1049 & 15,344 & 1351,1 & 0,2056 & Grande \\
\hline
\end{tabular}

Assim como no caso anterior, os filtros de Fourier modificados apresentaram as menores oscilações da resposta de magnitude e o filtro cosseno, da resposta de fase. O último também apresentou o menor overshoot da resposta de magnitude, porém, pelo mesmo motivo citado anteriormente, os filtros de Fourier modificados o superam, já que o overshoot desses ocorre antes do overshoot do filtro cosseno (pouco mais de um ciclo). Quanto à velocidade de convergência, os filtros de Fourier modificados são os únicos que convergem para quaisquer constantes de tempo.

\subsubsection{Sinais com harmônicas e ruídos aleatórios}

O objetivo deste tipo de teste é analisar o comportamento dos filtros na presença de ruídos. A razão entre o máximo ruído e a magnitude da componente de frequência fundamental e cada uma das componentes harmônicas é $2 \%$, conforme Lee et al. (2008). O sinal de teste utilizado é dado por:

$$
\begin{array}{r}
i_{3}(t)=\left[\sum_{n=1}^{30} \frac{50}{n} \cos (n \omega t+n \alpha)\right]-55 \cos \alpha \cdot e^{-\frac{t}{\tau}} \\
+5 \cos \alpha \cdot e^{-\frac{t}{\tau_{s}}}+\text { ruidos }
\end{array}
$$

Tabela 4: Índices médios de desempenho para o sinal de

\begin{tabular}{|c|c|c|c|c|c|c|c|}
\hline Filtro & $\mathbf{N}$ & $M\left(I D_{1}\right)$ & $M\left(I D_{2}\right)$ & $M\left(I D_{3}\right)$ & $M\left(I D_{4}\right)$ & $M\left(I D_{5}\right)$ & $M\left(I D_{6}\right)$ \\
\hline \multicolumn{8}{|c|}{$\alpha=4^{\circ}$} \\
\hline \multirow{3}{*}{$\mathbf{A}$} & 64 & 0,1938 & 0,1607 & 8,9230 & 300,00 & Grande & Grande \\
\hline & 96 & 0,1942 & 0,1607 & 8,9219 & 300,00 & Grande & Grande \\
\hline & 120 & 0,1944 & 0,1606 & 8,9209 & 300,00 & Grande & Grande \\
\hline \multirow{3}{*}{ B } & 64 & 0,2078 & 0,0064 & 2,3000 & 300,00 & Grande & Grande \\
\hline & 96 & 0,1960 & 0,0053 & 2,3127 & 300,00 & Grande & Grande \\
\hline & 120 & 0,1915 & 0,0049 & 2,3267 & 300,00 & Grande & Grande \\
\hline \multirow{3}{*}{ C } & 64 & 0,0363 & 0,9483 & 12,596 & 299,88 & 0,1406 & 0,2005 \\
\hline & 96 & 0,0828 & 0,7586 & 31,812 & 300,07 & 0,1476 & 0,1910 \\
\hline & 120 & 0,0620 & 0,8569 & 26,631 & 299,98 & 0,1444 & 0,1903 \\
\hline \multirow{3}{*}{ D } & 64 & 0,0363 & 0,9483 & 12,596 & 300,00 & 0,1406 & 0,2005 \\
\hline & 96 & 0,0828 & 0,7586 & 31,812 & 300,00 & 0,1476 & 0,1910 \\
\hline & 120 & 0,0620 & 0,8569 & 26,631 & 300,00 & 0,1444 & 0,1903 \\
\hline
\end{tabular}
teste da equação 37 para $\alpha=45^{\circ}$.

A Figura 4 apresenta sinais de teste, baseados na equação (38). Os valores de $\tau$ mostrados na Figura 4 são as constantes de tempo primárias.

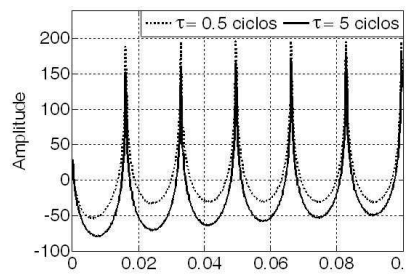

(a) $\alpha=10^{\circ}$

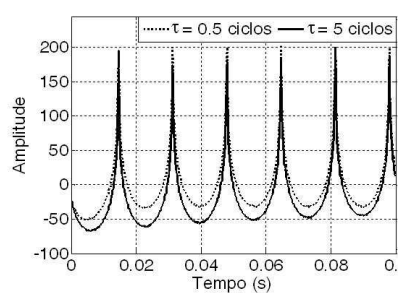

(b) $\alpha=45^{\circ}$
Figura 4: Sinais de teste contendo harmônicas até a ordem 30 , duas componente $\mathrm{DC}$ e ruídos.

As respostas dos filtros, no domínio do tempo, para frequiência de amostragem de $3840 \mathrm{~Hz}$ e $\alpha=10^{\circ}$ e $45^{\circ}$, são mostradas nas Tabelas 5 e 6 , respectivamente.

A inserção de ruídos nos sinais de teste causou um aumento nos índices que avaliam o desempenho dos filtros, o que já era esperado. A alteração mais notável diz respeito à convergência, tanto da resposta de magnitude como de fase. Analisando os índices de desempenho, nota-se que nenhum dos filtros converge, independente do valor da constante de tempo do sinal. No entanto, os filtros de Fourier modificados são os que apresentam respostas mais próximas do valor desejado.

Os filtros de Fourier modificados apresentaram as menores oscilações da resposta de magnitude e o filtro cosseno, da 
Tabela 5: Índices médios de desempenho para o sinal de teste da equação (38) para $\alpha=10^{\circ}$.

\begin{tabular}{|c|c|c|c|c|c|c|c|}
\hline Filtro & $\mathbf{N}$ & $M\left(I D_{1}\right)$ & $M\left(I D_{2}\right)$ & $M\left(I D_{3}\right)$ & $M\left(I D_{4}\right)$ & $M\left(I D_{5}\right)$ & $M\left(I D_{6}\right)$ \\
\hline \multicolumn{8}{|c|}{$\alpha=10^{\mathbf{o}}$} \\
\hline \multirow{3}{*}{ A } & 64 & 0,3153 & 0,2757 & 10,380 & 1449,9 & Grande & Grande \\
\hline & 96 & 0,3147 & 0,2715 & 11,244 & 1461,3 & Grande & Grande \\
\hline & 120 & 0,3183 & 0,2709 & 11,456 & 1468,0 & Grande & Grande \\
\hline \multirow{3}{*}{ B } & 64 & 0,1806 & 0,0100 & 3,8682 & 1700,0 & Grande & Grande \\
\hline & 96 & 0,1502 & 0,0084 & 4,4945 & 1700,0 & Grande & Grande \\
\hline & 120 & 0,1470 & 0,0076 & 4,5987 & 1700,0 & Grande & Grande \\
\hline \multirow{3}{*}{ C } & 64 & 0,0486 & 0,0437 & 8,6187 & 1301,0 & Grande & Grande \\
\hline & 96 & 0,0456 & 0,1864 & 13,116 & 1340,8 & Grande & Grande \\
\hline & 120 & 0,0455 & 0,1258 & 15,248 & 1351,5 & Grande & Grande \\
\hline \multirow{3}{*}{ D } & 64 & 0,0486 & 0,0437 & 8,6187 & 1301,0 & Grande & Grande \\
\hline & 96 & 0,0456 & 0,1864 & 13,116 & 1340,8 & Grande & Grande \\
\hline & 120 & 0,0455 & 0,1258 & 15,248 & 1351,5 & Grande & Grande \\
\hline
\end{tabular}

resposta de fase. Quanto ao overshoot, os filtros modificados também apresentaram vantagens, uma vez que o filtro cosseno possui um atraso de resposta maior.

Tabela 6: Índices médios de desempenho para o sinal de teste da equação 38 para $\alpha=45^{\circ}$.

\begin{tabular}{|c|c|c|c|c|c|c|c|}
\hline Filtro & $\mathbf{N}$ & $M\left(I D_{1}\right)$ & $M\left(I D_{2}\right)$ & $M\left(I D_{3}\right)$ & $M\left(I D_{4}\right)$ & $M\left(I D_{5}\right)$ & $M\left(I D_{6}\right)$ \\
\hline \multicolumn{8}{|c|}{$\alpha=\mathbf{4 5}^{\mathbf{0}}$} \\
\hline \multirow{3}{*}{$\mathbf{A}$} & 64 & 0,1989 & 0,1637 & 7,8770 & 300,00 & Grande & Grande \\
\hline & 96 & 0,1977 & 0,1620 & 9,1253 & 300,00 & Grande & Grande \\
\hline & 120 & 0,1953 & 0,1611 & 8,8303 & 300,00 & Grande & Grande \\
\hline \multirow{3}{*}{ B } & 64 & 0,0264 & 0,0064 & 1,2981 & 300,00 & Grande & Grande \\
\hline & 96 & 0,1939 & 0,0058 & 2,5173 & 300,00 & Grande & Grande \\
\hline & 120 & 0,1941 & 0,0050 & 2,2576 & 300,00 & Grande & Grande \\
\hline \multirow{3}{*}{ C } & 64 & 0,0601 & 0,9644 & 11,508 & 300,00 & Grande & Grande \\
\hline & 96 & 0,1016 & 11,057 & 32,073 & 300,00 & Grande & Grande \\
\hline & 120 & 0,0764 & 11,053 & 26,751 & 299,96 & Grande & Grande \\
\hline \multirow{3}{*}{ D } & 64 & 0,0601 & 0,9644 & 11,508 & 299,99 & Grande & Grande \\
\hline & 96 & 0,1016 & 11,057 & 32,073 & 300,00 & Grande & Grande \\
\hline & 120 & 0,0764 & 11,053 & 26,751 & 300,00 & Grande & Grande \\
\hline
\end{tabular}

Os diversos testes realizados permitiram classificar os filtros de acordo com os seus desempenhos. A Tabela 7 ordena os filtros de acordo cada um dos índices de desempenho considerados.

É possível concluir que, independente do sinal de entrada, os filtros de Fourier modificados apenas não obtiveram o melhor desempenho no que diz respeito ao overshoot do módulo $\left(\mathrm{M}\left(\mathrm{ID}_{3}\right)\right)$ e à oscilação do ângulo $\left(\mathrm{M}\left(\mathrm{ID}_{2}\right)\right)$, pois o filtro Cosseno foi melhor.

A questão do overshoot da resposta de magnitude já foi discutida anteriormente, e chegou-se a conclusão que, mesmo que o filtro cosseno apresente índices menores, os filtros de Fourier modificados possuem melhor desempenho, uma vez que os seus overshoots ocorrem antes do overshoot do filtro cosseno.

Além disso, existe também o fato da duração do overshoot. Os relés temporizados, por exemplo, aguardam um certo tempo antes de operarem, de modo a haver uma maior certeza da ocorrência da falta. Assim, mesmo que o valor do overshoot seja alto, a ponto de caracterizar uma falta, sua duração pode ser menor do que o tempo de atraso do relé, fazendo com que o mesmo não opere.

Tabela 7: Ordem de desempenho dos filtros por categoria, considerando 64 amostras por ciclo.

\begin{tabular}{|c|c|c|c|c|c|c|c|}
\hline \multirow{4}{*}{$\begin{array}{c}\mathbf{M}(\mathrm{ID}) \\
\mathbf{M}\left(\mathrm{ID}_{1}\right)\end{array}$} & \multirow{4}{*}{$\begin{array}{l}10 \\
45\end{array}$} & \multicolumn{3}{|c|}{$1^{\circ}$} & \multicolumn{2}{|c|}{$2^{\circ}$} & \multirow[t]{2}{*}{$3^{\circ}$} \\
\hline & & \multicolumn{5}{|c|}{ Sinal de teste: 30 harmônicas + 1 DC } & \\
\hline & & C/D & 0,0173 & B & 0,1495 & $\mathbf{A}$ & 0,2691 \\
\hline & & C/D & 0,0327 & $\mathbf{A}$ & 0,1657 & B & 0,3328 \\
\hline \multirow{2}{*}{$\mathbf{M}\left(\mathbf{I D}_{2}\right)$} & 10 & B & 0,0083 & $\mathrm{C} / \mathrm{D}$ & 0,0262 & $\mathbf{A}$ & 0,2354 \\
\hline & 45 & B & 0,0053 & $\mathbf{A}$ & 0,1390 & $\mathrm{C} / \mathrm{D}$ & 0,9205 \\
\hline \multirow{2}{*}{$\mathbf{M}\left(\mathbf{I D}_{3}\right)$} & 10 & B & 4,6419 & C/D & 9,1233 & $\mathbf{A}$ & 10,4280 \\
\hline & 45 & B & 2,1653 & $\mathbf{A}$ & 8,2091 & C/D & 12,1820 \\
\hline \multirow[b]{2}{*}{$\mathbf{M}\left(\mathbf{I D}_{4}\right)$} & 10 & C/D & 1298,6000 & $\mathbf{A}$ & 1446,3000 & B & 1700,0000 \\
\hline & 45 & C & 299,9000 & $\mathbf{A} / \mathbf{B} / \mathbf{D}$ & 300,000 & & \\
\hline \multirow{2}{*}{$\mathbf{M}\left(\mathbf{I D}_{5}\right)$} & 10 & $\mathrm{C} / \mathrm{D}$ & 0,2031 & $\mathbf{A} / \mathbf{B}$ & Grande & & \\
\hline & 45 & C/D & 0,1406 & $\mathbf{A} / \mathbf{B}$ & Grande & & \\
\hline \multirow{3}{*}{$\mathbf{M}\left(\mathbf{I D}_{6}\right)$} & 10 & C/D & 0,2708 & $\mathbf{A} / \mathbf{B}$ & Grande & & \\
\hline & 45 & C/D & 0,1979 & $\mathbf{A} / \mathbf{B}$ & Grande & & \\
\hline & \multicolumn{7}{|c|}{ Sinal de teste: $\mathbf{3 0}$ harmônicas + 2 DC } \\
\hline \multirow{2}{*}{$\mathbf{M}\left(\mathrm{ID}_{1}\right)$} & 10 & C/D & 0,0203 & B & 0,1547 & $\mathbf{A}$ & 0,3139 \\
\hline & 45 & C/D & 0,0363 & A & 0,1938 & B & 0,2078 \\
\hline \multirow{2}{*}{$\mathbf{M}\left(\mathbf{I D}_{2}\right)$} & 10 & B & 0,0099 & $\mathrm{C} / \mathrm{D}$ & 0,0290 & $\mathbf{A}$ & 0,2713 \\
\hline & 45 & B & 0,0064 & $\mathbf{A}$ & 0,1607 & C/D & 0,9483 \\
\hline \multirow{2}{*}{$\mathbf{M}\left(\mathbf{I D}_{3}\right)$} & 10 & B & 4,7487 & C/D & 9,6042 & $\mathbf{A}$ & 11,3230 \\
\hline & 45 & B & 2,3000 & A & 8,9230 & $\mathrm{C} / \mathrm{D}$ & 12,5960 \\
\hline \multirow[b]{2}{*}{$\mathbf{M}\left(\mathrm{ID}_{4}\right)$} & 10 & $\mathrm{C} / \mathrm{D}$ & 1297,8000 & $\mathbf{A}$ & 1444,6000 & B & 1700,0000 \\
\hline & 45 & C & 299,8800 & $\mathbf{A} / \mathbf{B} / \mathbf{D}$ & 300,0000 & & \\
\hline \multirow[b]{2}{*}{$\mathbf{M}\left(\mathbf{I D}_{5}\right)$} & 10 & C/D & 0,2031 & $\mathbf{A} / \mathbf{B}$ & Grande & & \\
\hline & 45 & C/D & 0,1406 & $\mathbf{A} / \mathbf{B}$ & 300,0000 & & \\
\hline \multirow[b]{2}{*}{$\mathbf{M}\left(\mathbf{I D}_{6}\right)$} & 10 & C/D & 0,7266 & $\mathbf{A} / \mathbf{B}$ & Grande & & \\
\hline & 45 & C/D & 0,2005 & $\mathbf{A} / \mathbf{B}$ & Grande & & \\
\hline \multicolumn{8}{|c|}{ Sinal de teste: 30 harmônicas + 2 DC + ruídos } \\
\hline \multirow{2}{*}{$\mathbf{M}\left(\mathbf{I D}_{1}\right)$} & 10 & C/D & 0,0486 & B & 0,1806 & $\mathbf{A}$ & 0,3153 \\
\hline & 45 & B & 0,0264 & C/D & 0,0601 & $\mathbf{A}$ & 0,1989 \\
\hline \multirow{2}{*}{$\mathbf{M}\left(\mathbf{I D}_{2}\right)$} & 10 & B & 0,0100 & $\mathbf{C} / \mathbf{D}$ & 0,0437 & $\mathbf{A}$ & 0,2757 \\
\hline & 45 & B & 0,0064 & $\mathbf{A}$ & 0,1637 & $\mathrm{C} / \mathrm{D}$ & 0,9644 \\
\hline \multirow{2}{*}{$\mathbf{M}\left(\mathbf{I D}_{3}\right)$} & 10 & B & 3,8682 & $\mathrm{C} / \mathrm{D}$ & 8,6187 & $\mathbf{A}$ & 10,3800 \\
\hline & 45 & B & 1,2981 & $\mathbf{A}$ & 7,8770 & C/D & 11,5080 \\
\hline \multirow{2}{*}{$\mathbf{M}\left(\mathbf{I D}_{4}\right)$} & 10 & C/D & 1301,0000 & A & 1449,9000 & B & 1700,0000 \\
\hline & 45 & D & 299,9900 & $\mathrm{~A} / \mathbf{B} / \mathbf{C}$ & 300,0000 & & \\
\hline \multirow{2}{*}{$\mathbf{M}\left(\mathbf{I D}_{5}\right)$} & 10 & Todos & Grande & & & & \\
\hline & 45 & Todos & Grande & & & & \\
\hline \multirow{2}{*}{$\mathbf{M}\left(\mathbf{I D}_{6}\right)$} & 10 & Todos & Grande & & & & \\
\hline & 45 & Todos & Grande & & & & \\
\hline
\end{tabular}

Onde:

A - Filtro de Fourier de ciclo completo

$B$ - Filtro Cosseno

C-Filtro de Fourier modificado proposto por Lee et al. (2008)

D - Filtro de Fourier modificado proposto por Sidhu et al. (2003)

Esse overshoot, de valor alto e duração pequena, é uma característica dos filtros de Fourier modificados, em 
contraste com o filtro Cosseno, que apresenta um overshoot de duração relativamente elevada, duração essa que poderia ser superior ao tempo de ajuste do relé, fazendo com que o mesmo operasse de maneira indevida.

Pela análise de todas as respostas dos filtros de Fourier modificados no domínio do tempo, notou-se que esses possuem a tendência de estimar valores elevados para os fasores da componente fundamental no momento em que a janela de dados se torna completa. No entanto, apenas cerca de 3 ou 4 amostras possuem valores altos, sendo que após esse tempo, a resposta se estabiliza. Uma vez que o tempo de atraso dos relés, geralmente, é superior a 4 amostras, esses altos valores não resultarão em má operação do relé.

Por fim, a Tabela 8 ordena os filtros considerando o desempenho global. Nota-se que os filtros de Fourier modificados foram os que obtiveram o melhor desempenho, seguidos dos filtros cosseno e Fourier de ciclo completo. $\mathrm{O}$ comportamento superior do filtro cosseno em relação ao de Fourier de ciclo completo também foi reportado por Tupiassú et al. (2003).

Tabela 8: Desempenho geral dos filtros

\begin{tabular}{cccc}
\hline \multirow{4}{*}{ Filtro } & \multicolumn{3}{c}{ Posição } \\
\cline { 2 - 4 } & $\mathbf{1}^{\mathbf{0}}$ & $\mathbf{2}^{\mathbf{0}}$ & $\mathbf{3}^{\mathbf{0}}$ \\
\cline { 2 - 4 } & $\begin{array}{c}\text { pourier modifi cado proposto } \\
\text { por Lee et al. (2008) } \mathbf{e} \\
\text { por Sidhu et al. (2003) }\end{array}$ & Cosseno & $\begin{array}{c}\text { Fourier de ciclo } \\
\text { completo }\end{array}$ \\
\hline
\end{tabular}

Os dois filtros de Fourier modificados apresentaram um desempenho semelhante em quase todos os casos simulados. Isso ocorreu devido ao ajuste do parâmetro $m$ no filtro modificado proposto por Sidhu et al. (2003). Os valores de $m$ escolhidos foram 31,47 e 59 para sinais amostrados em $3840 \mathrm{~Hz}, 5760 \mathrm{~Hz}$ e $7200 \mathrm{~Hz}$, respectivamente. Esses foram escolhidos por meio de simulações, sendo que para esses valores, o filtro apresentou o melhor desempenho. É interessante salientar que, se outros valores de $m$ tivessem sido escolhidos, os filtros teriam apresentado resultados diferentes e, conseqüentemente, desempenhos diferentes.

\subsection{Sinais obtidos a partir de simulações no programa ATP}

O sistema teste utilizado foi proposto por Yu \& Gu (2001), e é mostrado na Figura 5.

Foram simulados curtos-circuitos monofásicos e trifásicos, considerando resistências de falta de $18 \Omega$ e $1 \Omega$, respectivamente. $\mathrm{O}$ carregamento do sistema, o qual é alterado com a mudança do ângulo $\delta$, foi considerado como sendo $-10^{\circ}$. O ponto de ocorrência da falta é $10 \mathrm{~km}$ e a falta ocorre em t=16,7 ms. A Tabela 9 mostra os parâmetros de

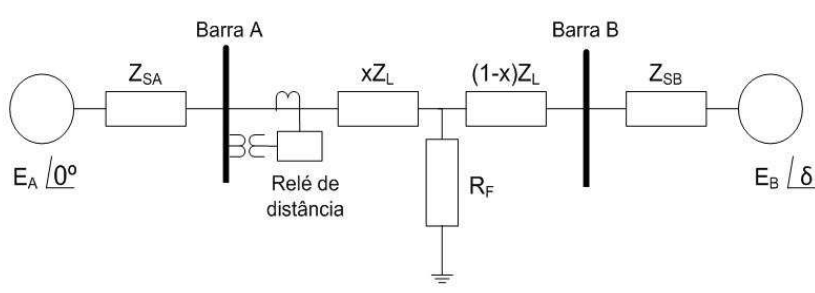

Figura 5: Sistema teste.

simulação do sistema.

Tabela 9: Parâmetros de simulação do sistema

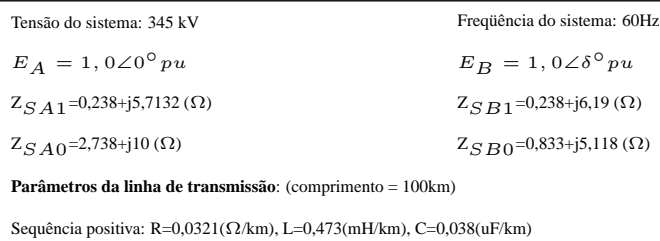

De acordo com as simulações anteriores, se concluiu que a amostragem de $3840 \mathrm{~Hz}$ é a mais vantajosa, portanto os sinais de teste obtidos do ATP foram amostrados nessa freqüência.

A Figura 6 apresenta o sinal de corrente resultante do curto-circuito trifásico.

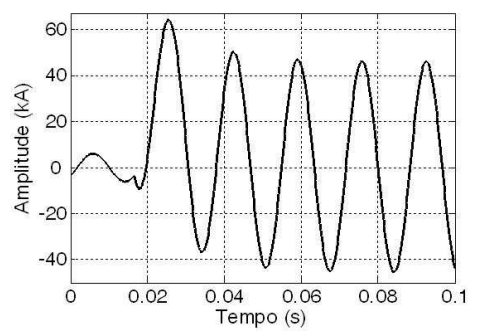

Figura 6: Sinal de teste obtido no ATP.

Os sinais foram pré-processados por um filtro passa-baixas Butterworth de $2^{\mathrm{a}}$ ordem e frequiência de corte de $480 \mathrm{~Hz}$. O parâmetro $m$ do filtro proposto por Sidhu et al. (2003) foi escolhido como sendo 31 .

A resposta dos filtros, no domínio do tempo, para o sinal da Figura 6 é mostrada na Figura 7.

Os resultados obtidos com sinais analíticos mostraram que os filtros de Fourier modificados obtiveram o melhor desempenho, seguidos pelo filtro cosseno. Esse fato é 
verificado novamente com os sinais de teste obtidos a partir do ATP.

A Figura 7 mostra que os filtros modificados apresentaram a melhor resposta para o sinal de corrente de curto-circuito trifásico, demonstrando, assim, a excelente capacidade de remover a componente DC.

As Figuras 7(c-d) mostram que os ruídos nas respostas dos filtros modificados ocorrem no intervalo de 1 a 2 ciclos. Dessa forma, esses ruídos não causarão nenhum problema de má operação do dispositivo de proteção. Pode-se concluir que, independente dos sinais de teste (analíticos ou obtidos por meio de simulações no ATP), os filtros modificados apresentaram o melhor desempenho, seguidos pelo filtro cosseno e TDFCC.

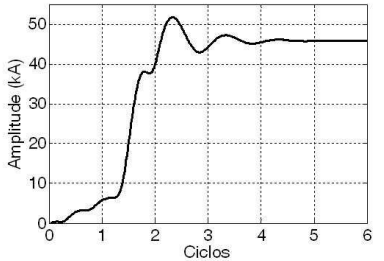

(a) $\alpha=10^{\circ}$

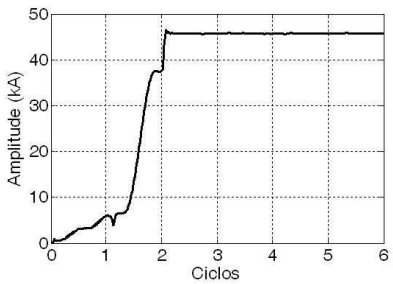

(c) $\alpha=10^{\circ}$

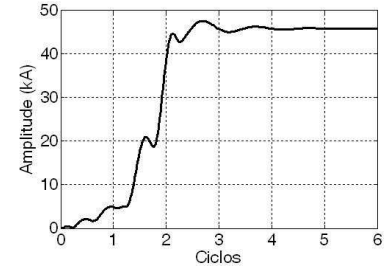

(b) $\alpha=45^{\circ}$

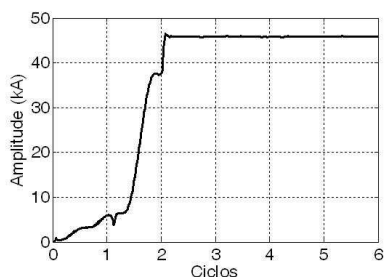

(d) $\alpha=45^{\circ}$
Figura 7: Resposta dos filtros. (a) TDFCC. (b) Cosseno. (c) TDF modificada proposta por Lee et al. (2008). (d) TDF modificada proposta por Sidhu et al. (2003).

\section{CONCLUSÕES}

Este artigo apresentou uma análise comparativa entre os algoritmos de filtragem mais comumente utilizados em relés digitais, juntamente com aqueles recentemente apresentados na literatura técnica.

Os filtros de Fourier modificados apresentaram o melhor desempenho. Esses possuem uma excelente capacidade de rejeição de harmônicas e da componente DC. Esses algoritmos são os que exigem maior esforço computacional, dado o grande número de operações matemáticas envolvidas. No entanto, esse fato não causou aumento significativo no tempo de simulação.

O filtro cosseno também apresenta boa capacidade de eliminar a componente DC. No entanto, é o filtro que fornece a resposta que implica em um maior atraso do dispositivo de proteção. O filtro TDFCC apresenta resposta com overshoot elevado e não é capaz de eliminar a componente DC, sendo que para constantes de tempo elevadas, a resposta se torna oscilatória.

Quanto à freqüência de amostragem dos sinais, as quais neste artigo foram consideradas como sendo $3840 \mathrm{~Hz}, 5760$ $\mathrm{Hz}$ e $7200 \mathrm{~Hz}$, conclui-se que as frequiências de $5760 \mathrm{~Hz}$ e $7200 \mathrm{~Hz}$, não trazem vantagens, uma vez que o desempenho dos filtros não apresenta melhora significativa, e ainda, o número de amostras a serem processadas aumenta, exigindo um relé de proteção com maior capacidade de processamento e, consequentemente, de custo mais elevado.

\section{REFERÊNCIA BIBLIOGRÁFICA}

Balamourougan, V. and Sidhu, T. S. (2006). A New Filtering Technique to Eliminate Decaying DC and Harmonics for Power System Phasor Estimation. IEEE Power India Conference.

Benmouyal, G. (1995). Removal of DC-Offset in Current Waveforms Using Digital Mimic Filtering. IEEE Transactions on Power Delivery, Vol.10, No.2, pp. 621-630.

Horowitz, S. and Phadke, A. (1995). Power System Relaying. Research Studies Press Ltd., England, $2^{\text {nd }}$ ed.

IEEE Tutorial Course (1979). Computer Relaying. 79p.

Lee. D., Kang, S., Nam, S. (2008). New Modified Fourier Algorithm to Eliminate the Effect of the DC Offset on Phasor Estimation Using DFT. IEEE Transmission and Distribution Conference and Exposition, 21-24 April.

Miranda, L. L. A (2005). Projetos de filtros digitais para análise de sinais do sistema elétrico. Dissertação de mestrado, UFRJ.

Phadke, A.G. and Thorp, J. S. (1988). Computer Relaying for Power Systems. Research Studies Press Ltd., England, 289 p.

Sachdev, M.S. and Baribeau, M.A. (1979). A new algorithm for digital impedance relays. IEEE Transactions on Power Apparatus and Systems, pp. 2232-2240.

Schweitzer III, E. O. and Hou, D. (1993). Filtering for Protective Relays. 47th Annual Georgia Tech Protective Relaying Conference, April 28-30. 
Sidhu, T. S., Zhang, X., Albarsi, F., Sachdev, M.S. (2003).

Discrete-Fourier-Transform-based Technique for

Removal of Decaying DC Offset from Phasor

Estimates. IEE Proc. Gener. Transm. Distrib., Vol.150,

No.6; pp. 745-752.

Stringer, N. T. (1997). The Effect of DC Offset on Current Operated Relays. IEEE Industrial and Commercial

Power Systems Technical Conference,

11-16 May, pp. 154 - 159.

Tupiassú, F. A., Bezerra, U. H., Santos Filho, O. G. (2003) Avaliação de algoritmos para proteção digital de sistemas elétricos de potência. In: XVII Seminário Nacional de Produção e Transmissão de Energia Elétrica. Uberlândia, MG.

Veloso, M. H. (2006). Índice de desempenho dos filtros digitais para proteção de sistemas elétricos de potência. Dissertação de mestrado, UFMG.

Wang, L. (1999). Frequency Responses of Phasor-based Microprocessor Relaying Algorithms. IEEE

Transactions on Power Delivery, Vol.14, No ${ }^{\circ} .1$, pp. 98-109.

Yu, S. and Gu, J. (2001). Removal of Decaying DC in Current and Voltage Signals Using a Modified Filter Algorithm. IEEE Transactions on Power Delivery, Vol.16, No.3; pp. 372-379. 